\title{
Crossbar Technique for the Failed Clavicular Hook Plate Fixation in an Acute Acromioclavicular Joint Dislocation: Salvage for Acromial Fracture after Clavicular Hook Plate
}

\author{
Kyoung Hwan Koh, Dong Ju Shin ${ }^{1 凶}$, Seong Mun Hwang ${ }^{1}$ \\ Department of Orthopaedic Surgery, Asan Medical Center, University of Ulsan College of Medicine, Seoul, ${ }^{1}$ Department of Orthopaedic Surgery, Daegu Fatima \\ Hospital, Daegu, Korea
}

\begin{abstract}
We experienced acromial erosion and subsequent fracture after the treatment of Rockwood type $\mathrm{V}$ acromioclavicular dislocation with hook plate and coracoclavicular ligament augmentation. It was treated by using a surgical technique to address an acromial fracture and subsequent losses of reduction in acromioclavicular joint with two trans-acromial cortical screws (crossbar technique). The reduction state of acromioclavicular joint could be maintained by these two screws. Our crossbar technique could be considered as a good salvage procedure for the reduction loss caused by cutout or significant erosion of acromion after insertion of clavicular hook plate.
\end{abstract}

(Clin Shoulder Elbow 2019;22(3):149-153)

Key Words: Acromioclavicular joint; Hook plate; Acromial fracture; Crossbar technique

More than 50 surgical techniques have been introduced to address clavicular joint dislocations." Among those numerous surgical techniques, the hook plate (Synthes, Oberdorf, Switzerland) is used widely because of the favorable clinical results without involving the acromioclavicular joint itself. ${ }^{2-6)}$ On the other hand, some authors reported complications, including acromial erosion or fracture caused by a hook plate. ${ }^{7-9)}$

The authors experienced an acromial erosion and subsequent fracture after the treatment of a Rockwood type $\mathrm{V}$ acromioclavicular dislocation with a hook plate and coracoclavicular ligament augmentation. This paper reports a surgical technique to address an acromial fracture and subsequent losses of reduction in an acromioclavicular joint with two trans-acromial cortical screws (crossbar technique).

\section{Case Report}

\section{Case 1}

A 69-year-old man with diabetes and osteoporosis, who had undergone surgery for colon cancer and was cured, was admitted with the complaint of right shoulder pain after falling from a bicycle. The radiographic findings indicated an acromioclavicular and coracoclavicular ligament injury of Rockwood type V (Fig. 1A) accompanied by multiple rib fractures. An open reduction and internal fixation of the acromioclavicular joint using a hook plate (Synthes) and coracoclavicular ligament augmentation using a $4.5 \mathrm{~mm}$ biodegradable screw (4.5 Bio-Corkscrew FT; Arthrex, Naples, FL, USA) was performed five days after the injury.

Open reduction and fixation of the acromioclavicular joint was carried out using a hook plate while monitoring the amount of reduction with a C-arm. A double-loaded $4.5 \mathrm{~mm}$ biodegradable screw was inserted into the coracoid base to augment the torn coracoclavicular ligament. One suture limb of one strand of suture was passed through the hole formed in the clavicle, and then tied with the opposite suture limb. The other strand of the suture was used to repair the coracoclavicular ligament. The postoperative radiographic findings revealed the maintained reduction of the acromioclavicular joint (Fig. 1B). A Kenny-How-

Received July 9, 2019. Revised August 1, 2019. Accepted August 4, 2019.

Correspondence to: Dong Ju Shin

Department of Orthopaedic Surgery, Daegu Fatima Hospital, 99 Ayang-ro, Dong-gu, Daegu 41199, Korea

Tel: +82-53-940-7320, Fax: +82-53-955-9990, E-mail: aabga@hanmail.net, ORCID: https://orcid.org/0000-0003-3612-3988

IRB approval: Daegu Fatima Hospital (No. DFH13ORIO214).

Financial support: None. Conflict of interests: None. 

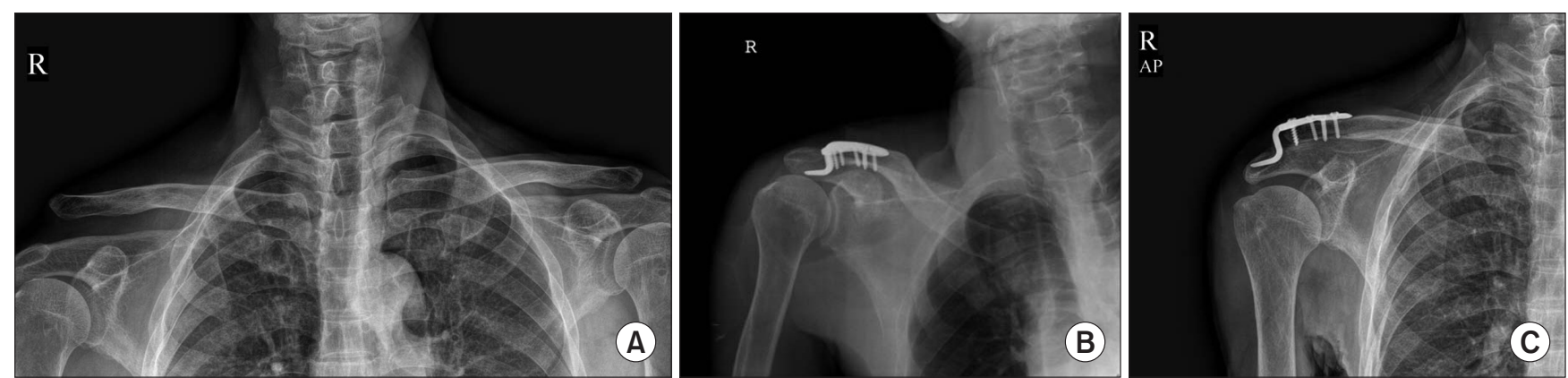

Fig. 1. (A) Right acromioclavicular joint showing a Rockwood type V acromioclavicular joint dislocation. (B) Reduced acromioclavicular joint after an open reduction and insertion of the clavicular hook plate. (C) Radiograph showing cutout of the hook through the acromion. AP: anteroposterior.

ard sling was applied postoperatively. Passive motion exercise was planned to begin at two weeks later and implant removal was scheduled at three months after surgery. The patient was discharged from hospital one week after surgery. He was allowed to perform daily activities and educated not to raise his arm over $90^{\circ}$. Plain radiographs at two weeks after surgery showed a maintained reduction and the patient did not complain of any rigorous pain. At five weeks, he visited the outpatient clinic with abrupt pain and claimed that a palpable protruding mass had been detected. A radiographic evaluation revealed an acromial fracture with the clavicular hook plate penetrating the acromion and reduction loss of the acromioclavicular joint (Fig. 1C).

During the second operation, cutout of the clavicular hook plate through the acromion was observed. After removing the clavicular hook plate, an acromial fracture and bony defect was were evident (Fig. 2A). Both the fractured acromion and dislocated acromioclavicular joint were addressed. First, two $3.0 \mathrm{~mm}$ countersinkable cannulated screws (Synthes) were applied to fix the acromial fracture under C-arm control (Fig. 2B) and hydroxyapatite (Bongros-HA; Daewoog, Korea) was applied to fill the bony defect. For acromioclavicular joint reduction, the same clavicular hook plate was re-inserted. Although there was a fracture and bony defect in the acromion, two cannulated screws crossing the fracture site of the acromion could be used as a buttress for the hook to prevent superior displacement of the clavicular hook plate (so-called 'cross-bar technique'; Fig. 2C). Subsequently, a locking compression plate (Synthes) and steel wire, which was passed through the cannulated screw, was used to support the fixation of the acromion (Fig. 2D, E). Fixation of the acromion and clavicle were confirmed after surgery (Fig. 2F). Two weeks after the reoperation, passive motion exercise was initiated, and strengthening exercise was begun after six weeks. The symptoms improved and the acromioclavicular joint was maintained in place. Five months after surgery, the hook plate was removed. After the internal fixator was removed, the followup X-ray showed no displacement of the acromioclavicular joint. At a final follow-up of 14 months after the second operation, range-of-motion was $160^{\circ}$ in forward elevation, $80^{\circ}$ in external rotation, and T6 in internal rotation (Fig. 2G). The clinical scores showed a pain visual analogue scale of 2, American Shoulder and Elbow Surgeons (ASES) score of 81.65, and Disabilities of the Arm, Shoulder, and Hand (DASH) score of 11.67.

\section{Case 2}

A 67-year-old female with no previously known disease was admitted for right shoulder pain after a fall. The radiographs showed Rockwood type V acromioclavicular dislocation. Open reduction and internal fixation were performed with a hook plate and the coracoclavicular ligament was repaired using the same technique described in case 1 . The postoperative radiograph showed a reduced acromioclavicular joint. A Kenny-Howard sling was applied and passive motion exercise was begun two weeks after surgery. At three weeks after surgery, the patient complained of abrupt posterior shoulder pain and the radiographic evaluation revealed significant penetration of the hook of the hook plate into the acromion (Fig. 3A). Secondary surgery was performed to prevent further penetration of the hook into the acromion and maintain the reduction of the acromioclavicular joint. During the second surgery, significant bone loss and penetration of the metal hook into the acromion was noted. Two trans-acromial cannulated screws were inserted crossing the injured acromion. These two screws were anticipated to be the buttress to prevent further cutout of a metal hook through the acromion and maintain the reduction of the acromioclavicular joint. Postoperative rehabilitation was performed in the same way as in case 1. Postoperatively, the posterior shoulder pain was improved gradually. The final radiograph showed the maintenance of reduction in the acromioclavicular joint and no further penetration of the metal hook (Fig. 3B, C).

\section{Discussion}

Although studies regarding the use of a hook plate reported favorable clinical results with a relatively easy surgical procedure, 

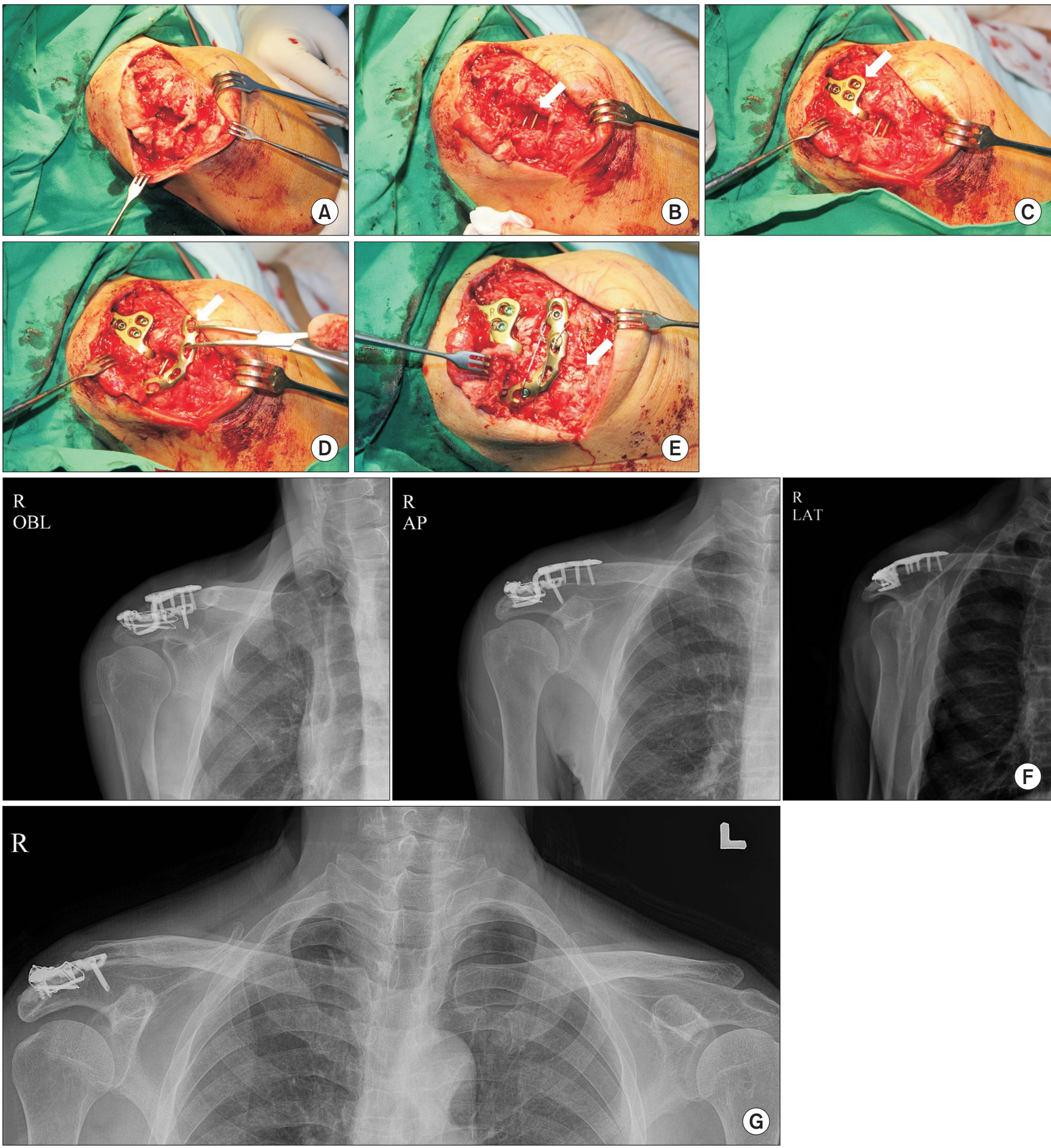

Fig. 2. Intraoperative photographs of the right shoulder in the sitting position. (A) Photograph showing a fracture and defect in the acromion after removing the clavicular hook plate during revision surgery. (B) Two cannulated screws (arrow) were inserted across the fracture site through the acromion. (C) The same clavicular hook plate (arrow) was re-inserted and the superior migration of the clavicular hook was prevented by two cannulated screws. (D) Locking compression plate (arrow) was applied to the acromial fracture site. (E) Screws were secured and the bone defect was filled with hydroxyapatite (arrow). (F) Radiographs taken after revision surgery showing a reduced acromioclavicular joint. (G) Radiograph taken 14 months after the second operation.

OBL: oblique, AP: anteroposterior, LAT: lateral. 

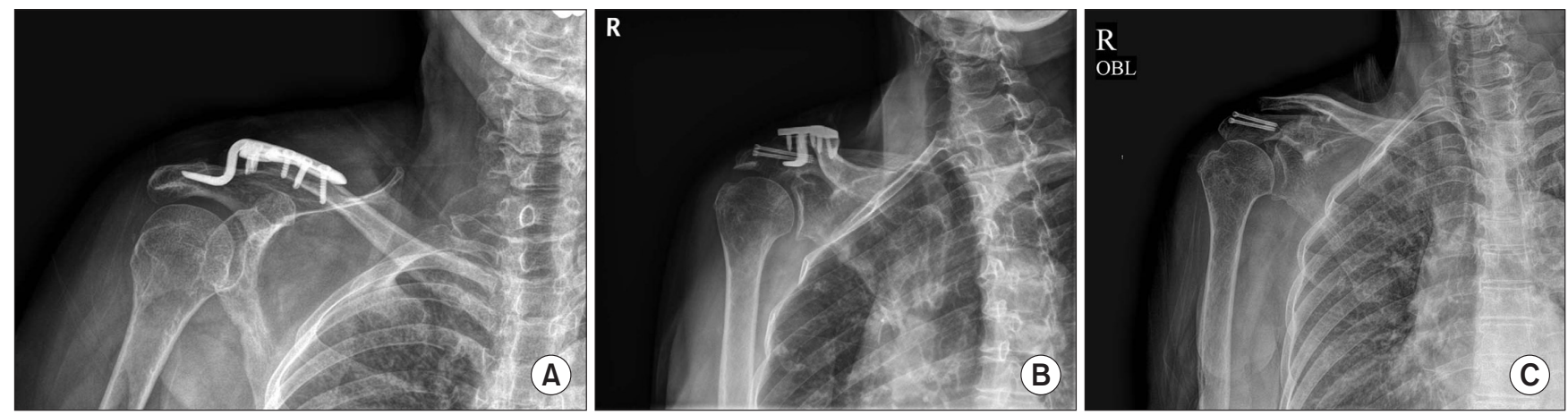

Fig. 3. (A) Radiograph showing significant erosion of acromion without a fracture by a hook. (B) Radiograph taken eight months after the initial surgery. Preventive trans-acromial cannulated screws maintained the reduction of the acromioclavicular joint without further superior migration of the clavicular hook plate. (C) Radiograph taken 11 months after the implant removal operation.

OBL: oblique.

complications are not uncommon. Indeed, acromial erosion could be encountered after applying the clavicular hook plate, even though true acromial fracture is not common. ${ }^{4,8,9)}$ Hoffler and Karas ${ }^{9)}$ encountered acromial fracture and reduction loss, which was similar to the complications the present cases, and treated them with ligament reconstruction surgery. On the other hand, ligament reconstruction is more appropriate for a chronic injury of acromioclavicular and coracoclavicular ligaments. Furthermore, reconstruction of the ligament only might leave the acromial fracture unhealed with some bone loss that could lead to residual pain or disabilities. Chiang et al. ${ }^{10)}$ also reported similar acromial fracture and reduction loss of the acromioclavicular joint. In their report, the authors managed the patient conservatively without surgical intervention because the patient did not want require a surgical procedure. A cosmetic deformity as well as functional disabilities and pain might be a problem in their case.

The technique of inserting two cannulated screws crossing the fracture site (crossbar technique) could fix the acromial fracture. In addition, these screws could be a buttress to prevent superior migration of the hook. Therefore, the reduction state of the acromioclavicular joint could be maintained by these two screws and might result in indirect healing of both the acromioclavicular and coracoclavicular ligament.

The crossbar technique could be used in cases with significant erosion of the acromion without cutout by the hook, as in the case 2. Preventive insertion of the trans-acromial screws could maintain the reduction of the acromioclavicular joint without any concerns of further erosion or superior migration of clavicular hook.

In conclusion, the crossbar technique could be considered a good salvage procedure for the reduction loss caused by cutout or significant erosion of the acromion after insertion of the clavicular hook plate.

\section{References}

1. Sim E, Schwarz N, Höcker K, Berzlanovich A. Repair of complete acromioclavicular separations using the acromioclavicular-hook plate. Clin Orthop Relat Res. 1995;(314):134-42.

2. De Baets T, Truijen J, Driesen R, Pittevils T. The treatment of acromioclavicular joint dislocation Tossy grade III with a clavicle hook plate. Acta Orthop Belg. 2004;70(6):515-9.

3. Ejam S, Lind T, Falkenberg B. Surgical treatment of acute and chronic acromioclavicular dislocation Tossy type III and V using the Hook plate. Acta Orthop Belg. 2008;74(4):441-5.

4. Eschler A, Gradl G, Gierer P, Mittlmeier T, Beck M. Hook plate fixation for acromioclavicular joint separations restores coracoclavicular distance more accurately than PDS augmentation, however presents with a high rate of acromial osteolysis. Arch Orthop Trauma Surg. 2012;132(1):33-9. doi: 10.1007/ s00402-011-1399-x.

5. Gstettner C, Tauber M, Hitzl W, Resch H. Rockwood type III acromioclavicular dislocation: surgical versus conservative treatment. J Shoulder Elbow Surg. 2008;17(2):220-5. doi: 10.1016/j.jse.2007.07.017.

6. Kienast B, Thietje R, Queitsch C, Gille J, Schulz AP, Meiners J. Mid-term results after operative treatment of rockwood grade III-V acromioclavicular joint dislocations with an AC-hookplate. Eur J Med Res. 2011;16(2):52-6. doi: 10.1186/2047783x-16-2-52.

7. Choi JY, Kim E, Jeong HJ, et al. Clinical comparison of two types of hook plate in surgical treatment of acromioclavicular dislocation: AO hook plate and Wolter plate. Clin Shoulder Elbow. 2012;15(2):123-9. doi: 10.5397/CiSE.2012.15.2.123.

8. ElMaraghy AW, Devereaux MW, Ravichandiran K, Agur AM. Subacromial morphometric assessment of the clavicle hook plate. Injury. 2010;41(6):613-9. doi: 10.1016/j.injury. 2009.12.012.

9. Hoffler CE, Karas SG. Transacromial erosion of a locked subacromial hook plate: case report and review of literature. J 
Shoulder Elbow Surg. 2010;19(3):e12-5. doi: 10.1016/j.jse. 2009.10.019.

10. Chiang CL, Yang SW, Tsai MY, Kuen-Huang Chen C. Acromion osteolysis and fracture after hook plate fixation for acromioclavicular joint dislocation: a case report. J Shoulder Elbow Surg. 2010;19(4):e13-5. doi: 10.1016/j.jse.2009.12.005. 